\title{
Effectiveness of psychological treatments for depression and alcohol use disorder delivered by community-based counsellors: two pragmatic randomised controlled trials within primary healthcare in Nepal
}

Mark J. D. Jordans, Nagendra P. Luitel, Emily Garman, Brandon A. Kohrt, Sujit D. Rathod, Pragya Shrestha, Ivan H. Komproe, Crick Lund and Vikram Patel

\section{Background}

Evidence shows benefits of psychological treatments in lowresource countries, yet few government health systems include psychological services.

\section{Aim}

Evaluating the clinical value of adding psychological treatments, delivered by community-based counsellors, to primary carebased mental health services for depression and alcohol use disorder (AUD), as recommended by the Mental Health Gap Action Programme (mhGAP).

\section{Method}

Two randomised controlled trials, separately for depression and AUD, were carried out. Participants were randomly allocated (1:1) to mental healthcare delivered by mhGAP-trained primary care workers (psychoeducation and psychotropic medicines when indicated), or the same services plus individual psychological treatments (Healthy Activity Program for depression and Counselling for Alcohol Problems). Primary outcomes were symptom severity, measured using the Patient Health

Questionnaire - 9 item (PHQ-9) for depression and the Alcohol Use Disorder Identification Test for AUD, and functional impairment, measured using the World Health Organization Disability Assessment Schedule (WHODAS), at 12 months post-enrolment.

\section{Results}

Participants with depression in the intervention arm $(n=60)$ had greater reduction in PHQ-9 and WHODAS scores compared with participants in the control $(n=60)(\mathrm{PHQ}-9$ : $\mathrm{M}=-5.90,95 \% \mathrm{Cl}$
-7.55 to $-4.25, \beta=-3.68,95 \% \mathrm{Cl}-5.68$ to $-1.67, P<0.001$, Cohen's $d=0.66$; WHODAS: $\mathrm{M}=-12.21,95 \% \mathrm{Cl}-19.58$ to -4.84 , $\beta=-10.74,95 \% \mathrm{Cl}-19.96$ to $-1.53, P=0.022$, Cohen's $d=0.42$ ). For the AUD trial, no significant effect was found when comparing control $(n=80)$ and intervention participants $(n=82)$.

\section{Conclusion}

Adding a psychological treatment delivered by communitybased counsellors increases treatment effects for depression compared with only mhGAP-based services by primary health workers 12 months post-treatment.

\section{Declaration of interest}

None.

\section{Keywords}

Psychological treatment; effectiveness; primary healthcare; lowand middle-income settings; Nepal.

\section{Copyright and usage}

(C) The Royal College of Psychiatrists 2019. This is an Open Access article, distributed under the terms of the Creative Commons Attribution-NonCommercial-NoDerivatives licence (http://creativecommons.org/licenses/by-nc-nd/4.0/), which permits noncommercial re-use, distribution, and reproduction in any medium, provided the original work is unaltered and is properly cited. The written permission of Cambridge University Press must be obtained for commercial re-use or in order to create a derivative work.
The global burden associated with mental disorders is high, especially for depression and alcohol use disorder (AUD). ${ }^{1}$ The vast majority of people with mental illness who live in low- and middle-income countries (LMICs) do not have access to adequate care, ${ }^{2}$ where only 1 person in every 27 with depression receives effective care. ${ }^{3}$ There is increasing evidence for the effectiveness of psychological treatments implemented by non-specialists in LMIC settings. ${ }^{4}$ Recent reviews demonstrate an effect size of 0.46 for depression outcomes in psychological treatments delivered by non-specialists in LMICs ${ }^{5}$ and promising outcomes for depression and AUD treatments by non-specialists health workers in primary and community healthcare in LMICs. ${ }^{6}$ Recent publications emphasise the need for research that assesses the efficacy of brief and flexible interventions that can be adapted to meet the needs of individuals across cultural contexts and employed to increase access to services. ${ }^{6,7}$ Specific psychological treatments - including motivational interviewing for AUD and behaviour activation techniques for depression - are recommended as first-line care by the
World Health Organization (WHO) Mental Health Gap Action Programme (mhGAP) Intervention Guide, a set of evidence-based clinical guidelines for non-specialist health workers and service providers to detect and treat mental illness. ${ }^{8}$ However, most mhGAP implementation in the government health systems of LMICs does not include these recommended specific psychological treatments because of a lack of time for primary healthcare workers to provide such care, the need for greater intensity training and supervision than standard 1-2 week mhGAP trainings and lack of patients' time for repeated weekly visits to primary care centres to receive the services. ${ }^{9}$ Therefore, alternative sustainable approaches for delivery of psychological treatments are needed. One proposed model to surmount this barrier is through the use of psychological service providers, for example counsellors, who can be deployed to deliver these psychological interventions in community settings as a complement to the diagnostic and medication management services provided through mhGAP-trained health workers in primary care facilities. Given the scarcity of resources in LMICs, we are interested 
in evaluating the feasibility of the same non-specialist service provider delivering psychological interventions in primary health and community settings for multiple disorders.

This study aimed to evaluate the effectiveness of adding community-based, counsellor-delivered psychological interventions for adults who initiated mhGAP-based primary care services for depression and AUD in Nepal.

\section{Method}

\section{Context: Programme for Improving Mental Health Care}

The Programme for Improving Mental Health Care (PRIME) aims to evaluate the feasibility, acceptability and outcomes of a multifaceted district level mental healthcare plan (MHCP) that targets the health facility, community and health system in five LMICs: Ethiopia, India, Nepal, South Africa and Uganda. ${ }^{10}$ The MHCP in Nepal has been described in detail previously. ${ }^{11}$ The programme was implemented in Chitwan, a predominantly rural district in southern Nepal. The total population of Chitwan was 579984 at the time of the study. The literacy rate of Chitwan district is $78.9 \%$, which is higher than the national average of $67 \% .{ }^{12}$ In the district of Nepal in which this trial took place, a representative community survey found that $11.2 \%$ of adults screened positive for depression and $5 \%$ screened positive for AUD. ${ }^{13}$ Among this group, treatment from primary care facilities was sought by $1.8 \%$ of those that screened positive for depression and $1.3 \%$ of those for AUD. ${ }^{12}$

The MHCP comprised interventions at the community, health facility and health service organisation levels. ${ }^{11}$ The community level included community sensitisation, proactive case detection ${ }^{14,15}$ and adherence support through home-based care. The facility-level packages included training and supervision for health workers to detect, diagnose and initiate treatment (i.e. emotional support, psychoeducation and psychotropic medication) for individuals with a diagnosis of a priority disorder, as well as referrals to specialised care following the mhGAP Intervention Guide. ${ }^{8}$ Finally, the health service organisation level included ensuring reliable supply of psychotropic medication, mechanisms for monitoring, capacity building and resource mobilisation. This package was available to all respondents enrolled in the study. The interventions delivered by primary care health workers are based on mhGAP guidelines and include assessment, psychoeducation, pharmacological treatments and initiating referral.

\section{Study design}

The two randomised controlled trials (RCTs) analysed here were nested in two treatment cohort studies, which have been described elsewhere in detail. ${ }^{16}$ Briefly, the primary objectives of the cohort studies were to evaluate the clinical-, social- and disability-related effects of treatment for depression or AUD. These treatments were initiated and delivered by health workers who were based in ten primary healthcare facilities in Chitwan, a district in southern Nepal. Adults (age $\geq 16$ years) with depression $(n=120)$ or with AUD $(n=162)$ were recruited upon diagnosis and followed up 3 and 12 months later. We included individuals that were recruited for the PRIME cohort studies of depression and AUD. Inclusion for the cohort study was determined by health worker diagnosis using the mhGAP guidelines for assessment and clinical decision making. Exclusion criteria for the depression and AUD cohorts were as follows: pregnancy, needing urgent medical treatment, diagnosis with psychosis or epilepsy (these patients were recruited for two different cohort studies) and being unable to communicate clearly. For the cohort studies, decision rules were established for the allocation to cohorts in case of comorbidity: psychosis or epilepsy took precedence over AUD, and AUD over depression. This meant that some of the AUD participants had also been diagnosed with depression as a secondary diagnosis $(n=3$, which is too small a subgroup to test for potential moderator effects). No changes to methods were made after commencement of the trials.

\section{Randomisation}

Among the participants who were already recruited into cohort studies evaluating mhGAP-based standards of care for depression or AUD, we individually randomised half to receive a psychological treatment (Healthy Activity Program $[\mathrm{HAP}]^{17}$ for depression and Counselling for Alcohol Problems [CAP] ${ }^{18}$ for AUD). After each participant completed their baseline assessments for the cohort study (without imposing a randomisation constraint), randomisation was done by the research coordinator in Kathmandu (N.P.L.) by using computer-generated random numbers (in SPSS Version 22 for Windows). A list of numbers (1-400) was randomised so that each number corresponded to either the treatment or control group. The ID code of each new eligible participant was sent to the research coordinator, who then matched it to the next number on the list. For those allocated to the treatment condition, the ID code was send to the study field coordinator and clinical supervisor so that they could connect these respondents to research assistant and community counsellors, respectively.

\section{Intervention service providers}

For the treatment arms of the trials, psychological treatments delivered by six community-based counsellors (four female, two male) were added. These counsellors were members of the local community who had completed at least a high-school education. The counsellors received a base training which included $400 \mathrm{~h}$ of classroom learning, $150 \mathrm{~h}$ of clinical supervision, $350 \mathrm{~h}$ of practice and $10 \mathrm{~h}$ of personal therapy spread out over 6 months. ${ }^{19}$ The communitybased counsellor training program has been used widely in the humanitarian non-governmental sector in Nepal for the past 15 years, and an estimated 400 community counsellors have been trained throughout the country during that time. In addition, for this study, the counsellors followed a 10 day combined training for $\mathrm{HAP}^{17}$ (for depression) and $\mathrm{CAP}^{18}$ (for AUD).

\section{Study interventions}

Participants in both study arms received the services that were part of the newly developed MHCP (standard of care), as described above. The control group participants therefore received mhGAPbased interventions (psychoeducation and pharmacological treatment when indicated) delivered by primary health workers. The participants in the treatment arm received additionally either HAP (for depression) or CAP (for AUD).

HAP is a manualised intervention that is delivered individually over 6-8 weekly sessions and consists of behavioural activation as the core therapeutic framework, which includes psychoeducation, behavioural assessment, activity monitoring, activity structuring, problem solving and activation of social networks. CAP is a manualised motivational activation intervention of four sessions delivered weekly, also delivered individually. It consists of detailed personal assessment, developing cognitive and behavioural skills (i.e. drink refusal skills, handling peer pressure, problem solving skills and handling difficult emotions) and managing potential relapses. These interventions have been developed through a systematic process of merging global evidence and contextually appropriate practices for treatment of depression and AUD. Recent trials in 
India have demonstrated the effectiveness and cost-effectiveness of $\mathrm{HAP}^{20,21}$ and $\mathrm{CAP}^{22,23}$ to reduce severity of symptoms and to increase remission. Both manuals were translated from the original language into Nepali by a bilingual Nepali psychologist, who had also received a training of trainers from the original developers. Bi-weekly supervision by the same trainer was delivered for the counsellors during the trials. The same service providers delivered both interventions within a routine primary healthcare setting.

\section{Outcome measures}

The primary outcomes were symptom severity and disability at 12 months follow-up. For the depression trial, symptom severity was assessed by the Patient Health Questionnaire - 9 item (PHQ-9), a self-reported screening tool designed for use in various medical settings. The PHQ-9 has been widely used and validated in primary care, medical out-patients and specialist medical services. ${ }^{24}$ The respondents are asked to score nine common symptoms of depression in the past 2 weeks. It has a 4-point rating scale from 0 'not at all' to 3 'always'. The PHQ-9 has been translated and validated in a primary care population in Chitwan, Nepal; with an area under the curve of 0.94 ( $95 \%$ CI $0.87-0.99$ ), sensitivity of 1.00 and specificity of 0.40 for a cut-off score of $\geq 5$ and a Cronbach's alpha of 0.84 in the validation sample. $^{25}$

For the AUD trial, symptom severity was assessing by the Alcohol Use Disorder Identification Test (AUDIT), developed by WHO as a screening tool for AUD in primary healthcare. ${ }^{26}$ The AUDIT has been validated in Nepal and a cut-off score of $\geq 9$ has been recommended as the threshold to identify probable alcohol dependence or alcohol misuse for both males (sensitivity 96.7, specificity 91.7) and females (sensitivity 94.3 , specificity 91.4), and has a Cronbach's alpha of $0.82 .^{27}$

In both depression and AUD samples, disability was assessed using the 12-item WHO Disability Assessment Schedule (WHODAS 2.0), measuring functional impairment over the previous 30 days. The instrument has been validated in a range of settings and cultures ${ }^{28}$ and has been previously used in Nepal. ${ }^{29,30}$ Cronbach's alpha for the WHODAS is 0.82 (depression trial participants) and 0.86 (AUD trial participants).

The secondary outcomes were response rates for depression, defined as at least 50\% reduction in PHQ-9 score compared with baseline, and reduction to low-risk drinking levels rates for AUD, defined as subthreshold AUDIT score (below nine). ${ }^{20,22}$ All instruments were administered as part of the parent cohort study, therefore no additional measures were included for the trials. In addition, we have included demographic measures (gender, age, marital status, religion, caste, education level and employment). We also collected process indicators (number of HAP and CAP sessions). No changes to outcomes were made after the start of the trials.

\section{Procedures}

Data collection was conducted by 12 research assistants who had completed at least an undergraduate degree. The research assistants received a 1 month training covering the topics of interviewing skills, rapport building, informed consent, ethical consideration, inclusion/exclusion criteria and content of the questionnaire. Data collection was conducted at baseline and follow-up measurements at 3 and 12 months after diagnosis and treatment initiation. Research assessors were blinded to participants' assignment to study arms; participants could not be blinded. Baseline assessments were initiated at the clinic and finalised in the participants' home within 3 days from initial contact. Written and oral information was provided to each of the study participants about the objectives and process of the study. Written informed consent was a requirement for enrolment into the trials. An adverse events reporting procedure was in place, including a brief protocol to assess suicidality. Serious adverse events were defined as death, suicide attempt and admission to hospital from any cause. Our strategy to limit loss to follow-up included: conducting up to three home visits, contacting by phone in case home visits were unsuccessful, visiting neighbouring districts in case respondents had migrated nearby and using a 4 and 8 week period for the assessments for the short term (midline) and long term (endline), respectively (2-4 weeks before and 2-4 weeks after the set date for assessments). A Data Safety Management Committee established by the Transcultural Psychosocial Organization Nepal with volunteer members external to the organisation (who had no involvement in the study funding, design or implementation) reviewed any reported adverse events. Ethical approval for the RCT was obtained from the Nepal Health Research Council (NHRC). The cohort studies were approved by the NHRC, and the ethics boards of the Faculty of Health Sciences, University of Cape Town, South Africa and WHO, Geneva, Switzerland. This study is registered with the International Standard Randomised Controlled Trials Number (ISRCTN) registry (ISRCTN72875710) (protocol available at: https://www.isrctn.com/).

\section{Statistical analyses}

Based on an estimated $80 \%$ power for the primary outcomes and a $10 \%$ loss to follow-up, two-sided alpha of 0.05 , we aimed to recruit 426 participants in both trials combined. However, the trials were embedded within a cohort study, so the sample size for the RCT reflects the number of people diagnosed and recruited in the cohort studies, therefore placing pragmatic constraints on recruitment for the randomised sample.

We first used non-parametric tests to compare baseline demographic and health-related characteristics between control and intervention participants, and between participants retained in the study and those lost to follow-up within each study arm; we used the Mann-Whitney U test for continuous variables and Fisher's exact test for categorical variables.

The midline and endline effects of HAP and CAP (for depression and AUD trials, respectively) were assessed by comparing the mean change in primary outcome scores from baseline between control and intervention participants in each trial. The differences in means and 95\% confidence intervals for the PHQ-9 and AUDIT (for depression and AUD trials, respectively) and for the WHODAS (both trials) were estimated by using unadjusted negative binomial regression, as none of the scores were normally distributed. For each trial, imbalances in baseline characteristics (Mann-Whitney or Fisher's $P<0.07$ ) were accounted for by using inverse probability weights: variables on which treatment and control arm participants differed were entered as covariates into a logistic regression model to calculate the probability of being in the treatment arm. The inverse of that probability was then used as a weight and included in the negative binomial regressions. ${ }^{31,32}$

Cohen's $d$ effect sizes were calculated on all primary outcomes as the mean difference in change in score from baseline to 3 months, or from baseline to 12 months, between control and intervention arms, divided by the pooled standard error. ${ }^{33,34}$

Secondary outcomes, the relative risk of reduction to low-risk drinking levels (for AUD trial, AUDIT $<9)$ and response $(\geq 50 \%$ reduction of PHQ-9 score for the depression trial) at 3 and 12 months were then assessed. The risk ratios and 95\% confidence intervals were estimated using log-binomial regression, again with inverse probability weights to account for baseline imbalances between the two arms. 
Finally, to assess the effect of psychological treatment on functioning (WHODAS) at 3 and 12 months post-recruitment among people affected by either of the two mental disorders, negative binomial regressions were conducted, this time combining samples from both trials. Again, any differences in baseline characteristics between control and treatment arms were accounted for by using inverse probability weights.

All outcome analyses were on an intention-to-treat basis with use of multiple imputation for those with missing outcome data. Continuous variables were imputed using Poisson models, whereas binary variables were imputed using logistic regression. Sensitivity analyses were conducted by running the same analyses among all participants with available data, without imputation.

\section{Results}

\section{Sample characteristics}

A total of 2044 individuals were approached for the cohort study, ${ }^{16}$ 312 met eligibility criteria of whom $9.6 \%$ did not consent to participate in the study. Of those who provided consent for the cohort study, 120 (5.9\%) received a diagnosis of depression and were enrolled in the depression trial and $162(7.9 \%)$ received a diagnosis of AUD and were enrolled in the AUD trial (see Fig. 1). Recruitment and baseline interviews took place between August 2014 and August 2015, and follow-up interviews occurred between December 2014 and August 2016. Reasons for loss to follow-up included migration $(n=34)$, unable to consent to participate $(n=9)$, unwilling to participate $(n=11)$, unavailable for interviews $(n=5)$ and death $(n=2)$.

Table 1 provides an overview of the demographic and clinical characteristics of participants recruited in each of the two samples, by arm. Despite the randomisation process, some differences in baseline demographic characteristics were found. Control and intervention arms differed $(P<0.07)$ in terms of education in the HAP trial and in terms of religion and social support in the CAP trial.

A total of 8 (13.3\%) participants in the depression intervention arm and 15 participants (18.8\%) in the AUD intervention arm did not receive any counselling sessions. In both depression and AUD intervention arms, the majority of participants received between three and five sessions (60.0 and $53.8 \%$, respectively). For the depression trial participants, the mean number of sessions was 3.8 (s.d. = 1.99) and the median was 4 (interquartile range 35). For the AUD trial the mean was 3.1 (s.d. =1.85) and the median was 3 (interquartile range 2-4) (although CAP is designed to be a four session intervention, in practice the content was at times spread out over more sessions as a result of shortened sessions due to limited availability of time among patients). See also Fig. 2.

In the AUD trial, 16 (19.5\%) participants in the control arm and $14(17.5 \%)$ in the intervention arm were lost to follow-up during the course of the study and did not complete the study. In the depression trial, this was the case for $9(15.0 \%)$ and 14 (23.3\%) participants in the control and intervention arm, respectively. In the majority of cases, in both trials (depression: $n=13,56.5 \%$; AUD: $n=16,53.3 \%$ ), this was due to participants migrating. There were no differences in baseline demographic or clinical characteristics between these two groups in the depression trial, although participants lost to follow-up had marginally greater AUDIT scores at baseline. In the AUD trial, participants retained in and lost to follow-up from the study differed in terms of religion and education. (See Supplementary Table 1 available at https://doi.org/10.1192/bjp. 2018.300).

\section{Depression trial outcomes}

The primary and secondary outcomes for the depression trial are presented in Table 2. At endline (12 month follow-up), the two arms differed significantly in change in PHQ-9 and WHODAS scores. At 12 months follow-up in the depression trial, participants in the HAP arm had a PHQ-9 score reduction of 9.6 (adjusted mean difference) compared with 5.9 in the control arm $(\beta=-3.7,95 \%$ CI -5.7 to $-1.7, P<0.001$ ). In the intervention (HAP) arm, the WHODAS score reduction was 22.9 (adjusted mean difference) compared with 12.2 in the control arm $(\beta=-10.7,95 \%$ CI -20.0 to $-1.5, P=0.022)$. Effect sizes for these changes at 12 months follow-up for symptom reduction were 0.66 (Cohen's $d$ ) and 0.42 for functioning impairment. See Table 2.

At 3 month follow-up in the depression trial, participants in the intervention (HAP) arm had an adjusted mean change in PHQ-9 score of -8.7 (95\% CI -9.95 to -7.4 ), compared with -5.9 (95\% CI -7.5 to -4.4 ) in the control group (95\% CI -4.8 to $-0.7, P=0.009)$. There was no significant difference in change in WHODAS scores between the two arms at the 3-month followup, however.

We see a significant between-group difference for response on the PHQ-9 at 12 months (risk ratio $1.42,95 \%$ CI $1.09-1.85$, $P=0.010)$. This difference was less pronounced at the 3 month follow-up (risk ratio $1.28,95 \%$ CI $0.97-1.69, P=0.076$ ).

\section{Outcomes AUD trial}

The primary and secondary results for the AUD trial are presented in Table 3. Results indicate that the reduction in AUDIT scores from baseline to the 12 month follow-up between the control $(\beta=-9.78$, $95 \% \mathrm{CI}-12.49$ to -7.08$)$ and intervention participants $(\beta=-11.948$, $95 \% \mathrm{CI}-14.80$ to -9.08$)$ did not differ significantly $(\beta=-2.16,95 \%$ $\mathrm{CI}-6.10$ to $1.79, P=0.285)$. Change in WHODAS scores from baseline to the 12-month follow-up also did not differ between the two arms $(\beta=-3.55,95 \%$ CI -11.16 to $4.06, P=0.360)$. Similarly we found no significant difference in the rate of reduction to low-risk drinking levels reported on the AUDIT (risk ratio 1.47, 95\% CI $0.87-2.48, P=0.150$ ). Sensitivity analyses conducted with available data show similar results for both the depression and AUD trials (see Supplementary Tables 2 and 3).

\section{Effects of interventions on WHODAS among participants with AUD or depression}

Results of the negative binomial regressions among combined AUD and depression trial participants demonstrate a small but significant effect size for change in WHODAS scores from baseline to endline in favour of the intervention arm $(\beta=-6.04,95 \% \mathrm{CI}$ -11.88 to $-0.20, P=0.042, d=0.24$ ), although changes from baseline to midline did not differ between control and intervention arms $(\beta=-3.53,95 \% \mathrm{CI}-9.40$ to $2.35, P=0.240, d=0.14)$.

\section{Discussion}

Psychological treatment (HAP) for depression delivered by community-based counsellors confers additional short- and long-term benefit compared with health worker-delivered, mhGAP-based standard of care (psychoeducation, pharmacological treatment) with outcomes of reduced depression symptoms and improved daily functioning. This means that for treatment of depression in a primary healthcare setting, there is evidence that adding a brief psychological treatment has added value, with moderate effect size, for short- and long-term outcomes.

This result is especially salient given: (a) the relatively small sample; (b) a comparator condition following evidence-based 


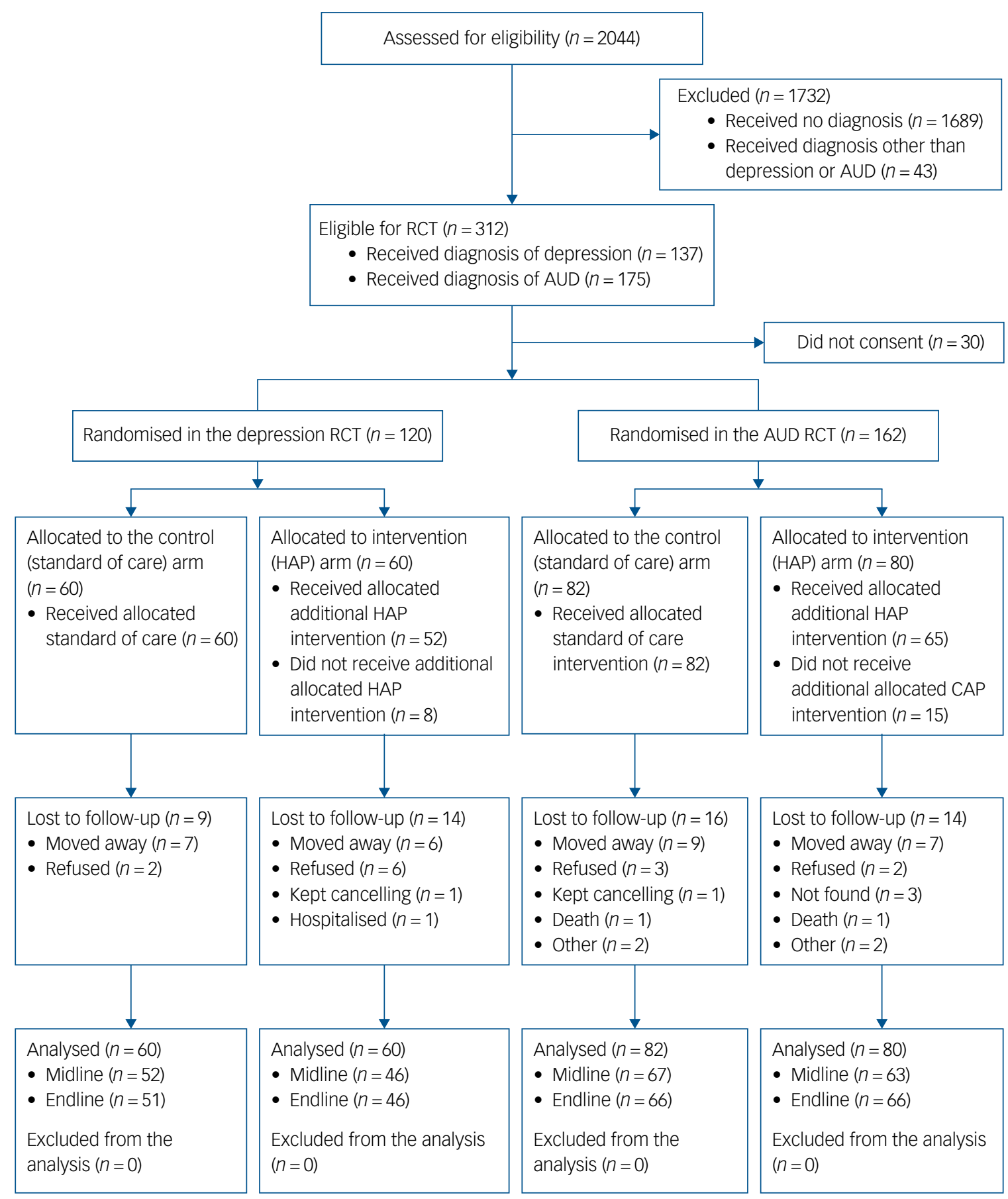

Fig. 1 Flow diagram of recruitment and follow-up process. AUD, alcohol use disorder; CAP, Counselling for Alcohol Problems; HAP, Healthy Activity Program; RCT, randomised controlled trial.

treatment guidelines, representing the current standard of improved mental healthcare following mhGAP; (c) pragmatic trial conditions including variance in attendance among participants in the treatment arm - therefore closely representing real-world implementation; and (d) the long-term effect at 12 month post-treatment given relapse rates for depression. The effect of the intervention on functioning is not seen at 3 months but only at 12 months, which may reflect the longer period needed for symptom reduction to contribute to improvement in daily functioning.

For AUD, we could not confirm the added value of a counsellordelivered psychological treatment over health worker-delivered mental healthcare alone (consisting of psychoeducation and pharmacological treatment when indicated) in reducing symptoms or improving functioning among individuals with AUD. We do see better results in the CAP arm, however, none of these reached statistical significance. This is contrary to the findings from the CAP trial in India. ${ }^{22,23}$ Given the lack of differences between the two arms, any correlates of change in AUDIT scores at 12 months would not have been attributable to the CAP intervention. The possible reasons for the absence of evidence for the added value of CAP are three-fold: First, it might be explained by the fact that the CAP is not designed for people with alcohol dependence and the original trial findings were restricted to participants with harmful drinking, ${ }^{22}$ whereas in the current study people with harmful alcohol use and alcohol dependence are combined to reflect actual practice. 
Table 1 Baseline characteristics of the participants recruited in each sample

\begin{tabular}{|c|c|c|c|c|c|c|c|c|c|c|}
\hline & \multicolumn{5}{|c|}{ Depression RCT $(N=120)$} & \multicolumn{5}{|c|}{ AUD RCT $(N=162)$} \\
\hline & \multirow[b]{2}{*}{$N$} & \multicolumn{2}{|c|}{$\begin{array}{l}\text { Standard of care } \\
\qquad(N=60)\end{array}$} & \multicolumn{2}{|c|}{$\begin{array}{c}\text { Standard of care + } \\
\operatorname{HAP}(N=60)\end{array}$} & \multirow[b]{2}{*}{ N } & \multicolumn{2}{|c|}{$\begin{array}{l}\text { Standard of care } \\
\qquad(N=82)\end{array}$} & \multicolumn{2}{|c|}{$\begin{array}{l}\text { Standard of care + CAF } \\
\qquad(N=80)\end{array}$} \\
\hline & & $N$ or med. & $\%$ or IQR & $N$ or med. & $\%$ or IQR & & $N$ or med. & $\%$ or IQR & $N$ or med. & $\%$ or IQR \\
\hline \multicolumn{11}{|l|}{ Gender } \\
\hline Male & 18 & 7 & 11.7 & 11 & 18.3 & 136 & 70 & 85.4 & 66 & 82.5 \\
\hline Female & 102 & 53 & 88.3 & 49 & 81.7 & 26 & 12 & 14.6 & 14 & 17.5 \\
\hline \multicolumn{11}{|l|}{ Age } \\
\hline $16-30$ & 27 & 9 & 15.0 & 18 & 30.0 & 21 & 11 & 13.4 & 10 & 12.5 \\
\hline $30-50$ & 61 & 31 & 51.7 & 30 & 50.0 & 98 & 45 & 54.9 & 53 & 66.3 \\
\hline$>50$ & 32 & 20 & 33.3 & 12 & 20.0 & 43 & 26 & 31.7 & 17 & 21.2 \\
\hline \multicolumn{11}{|l|}{ Marital status } \\
\hline No partner & 22 & 7 & 11.7 & 15 & 25.0 & 10 & 8 & 9.8 & 2 & 2.5 \\
\hline Has a partner & 98 & 53 & 88.3 & 45 & 75.0 & 152 & 74 & 90.2 & 78 & 97.5 \\
\hline \multicolumn{11}{|l|}{ Religion } \\
\hline Hindu & 102 & 51 & 85.0 & 51 & 85.0 & $123^{*}$ & $57 *$ & $69.5^{*}$ & $66^{*}$ & $82.5^{*}$ \\
\hline Other & 18 & 9 & 15.0 & 9 & 15.0 & $39 *$ & $25^{*}$ & $30.5^{*}$ & $14^{*}$ & $17.5^{*}$ \\
\hline \multicolumn{11}{|l|}{ Caste } \\
\hline Brahman/Chhetri & 48 & 21 & 35.0 & 27 & 45.0 & 56 & 28 & 34.2 & 28 & 35.0 \\
\hline Janajati & 35 & 21 & 35.0 & 14 & 23.3 & 41 & 26 & 31.7 & 15 & 18.8 \\
\hline Dalit & 29 & 15 & 25.0 & 14 & 23.3 & 47 & 20 & 24.4 & 27 & 33.7 \\
\hline Other & 8 & 3 & 5.0 & 5 & 8.3 & 18 & 8 & 9.8 & 10 & 12.5 \\
\hline \multicolumn{11}{|l|}{ Educational level } \\
\hline Uneducated/illiterate & $36^{*}$ & $22^{*}$ & $36.7 *$ & $14^{*}$ & $23.3^{*}$ & 37 & 18 & 22.0 & 19 & 23.8 \\
\hline Non-formal/less than primary school & $35^{*}$ & $21 *$ & $35.0^{*}$ & $14^{*}$ & $22.3^{*}$ & 30 & 17 & 20.7 & 13 & 16.2 \\
\hline Primary school and above & $49 *$ & $17^{*}$ & $28.3^{*}$ & $32^{*}$ & $53.3^{*}$ & 95 & 47 & 57.3 & 48 & 60.0 \\
\hline \multicolumn{11}{|l|}{ Employment } \\
\hline Not employed & 85 & 43 & 71.7 & 42 & 73.7 & 36 & 20 & 24.4 & 16 & 20.2 \\
\hline Employed & 32 & 17 & 28.3 & 15 & 26.3 & 125 & 62 & 75.6 & 63 & 79.8 \\
\hline \multicolumn{11}{|l|}{ Household food insecurity } \\
\hline No & 7 & 3 & 5.0 & 4 & 7.0 & 5 & 3 & 3.7 & 2 & 2.5 \\
\hline Yes & 110 & 57 & 95.0 & 53 & 93.0 & 156 & 79 & 96.3 & 77 & 97.5 \\
\hline PHQ-9 score & 120 & 12 & 5 & 13 & 4.5 & 162 & 9 & 7 & 7.5 & 6 \\
\hline AUDIT score & 120 & 0 & 1.5 & 0 & 0.5 & 162 & 26 & 9 & 27 & 9 \\
\hline WHODAS score & 120 & 38.9 & 33.3 & 34.7 & 22.2 & 162 & 18.1 & 19.4 & 19.4 & 26.4 \\
\hline OSS-3 score & 117 & 11 & 4 & 10 & 3 & $161^{*}$ & $10.5^{\star}$ & $3^{*}$ & $12^{\star}$ & $3^{*}$ \\
\hline
\end{tabular}

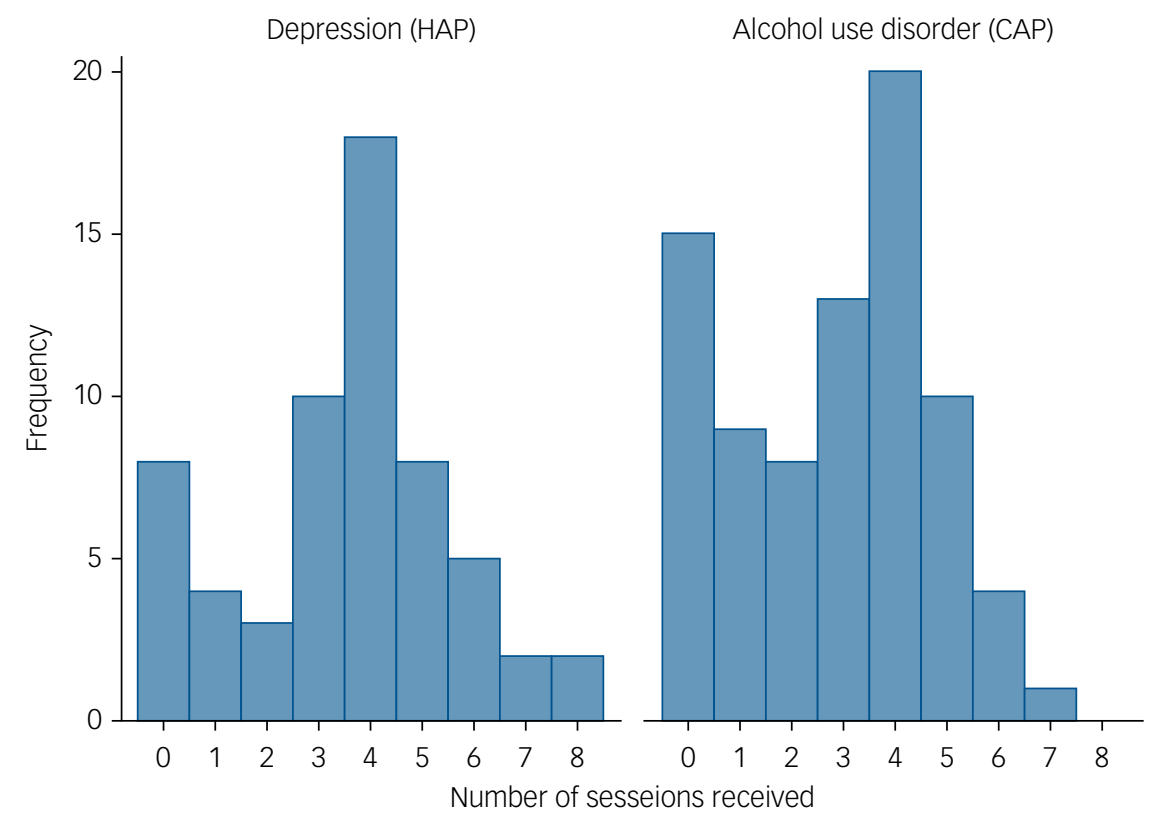

Fig. 2 Number of sessions per treatment arm. CAP, Counselling for Alcohol Problems; HAP, Healthy Activity Program. 


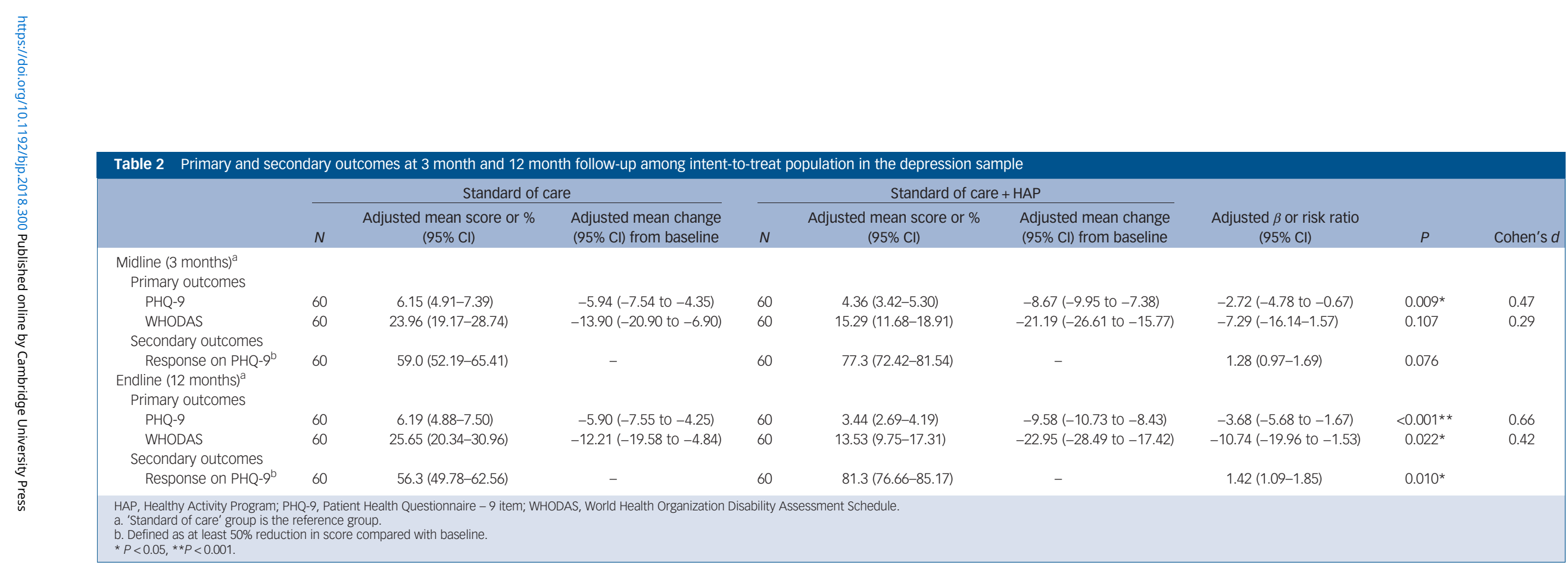

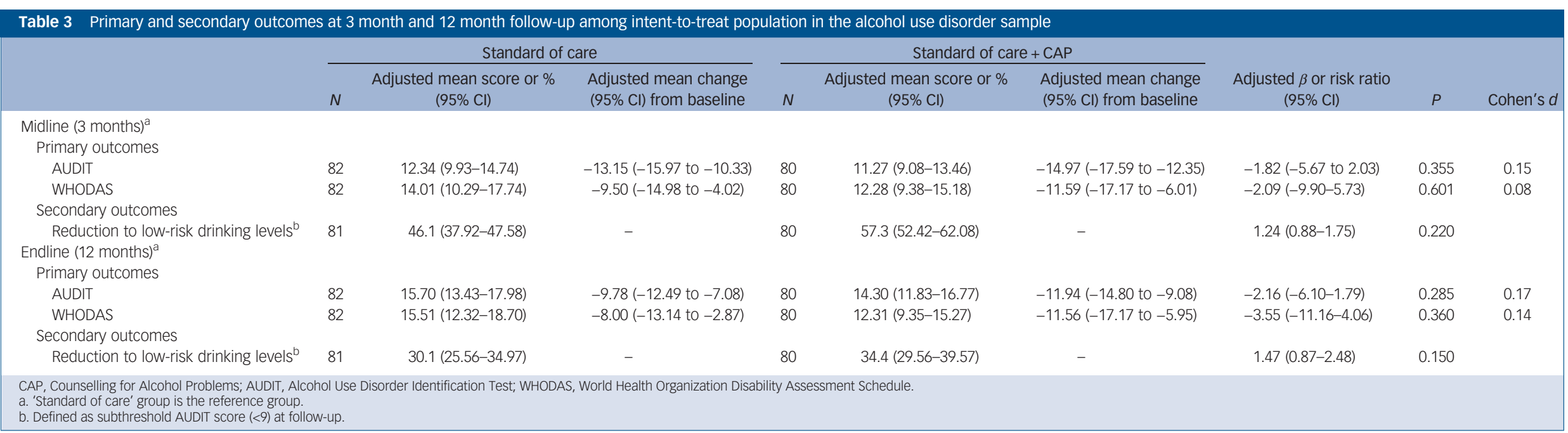


Second, the newly introduced mhGAP-based standard of care provided by the health workers is possibly sufficient, which is in line with evidence for the effectiveness of brief alcohol interventions within primary care. ${ }^{35}$ Another study in Nepal showed that the benefits for people with psychosis and epilepsy were no greater among those who received psychosocial services in addition to primary care services. ${ }^{36}$ It may be that for certain conditions, simply introducing primary care services carries most of the variance in improved outcomes.

Third, there could be a methodological explanation in that we may not have reached the sample for which the study was powered to detect statistically significant differences. The reason for this is because the trials were embedded within the larger cohort study, for which a time restriction for enrolment was applied following consortium guidance. Finally, although it is almost exclusively men receiving treatment for AUD, the majority of the counsellors were female. Gender matching in delivering CAP may be better suited for treatment engagement in Nepali society.

In addition, this study aimed to evaluate an integrated approach of delivering mental healthcare for multiple disorders by non-specialist providers. With regards to the outcome of functioning, perhaps the most salient patient-centred outcome, we see a significant improvement for those receiving treatment for depression or AUD at 12 months.

The study had several limitations. First, despite randomisation we did find baseline differences between randomised arms. These differences were statistically controlled for in the analyses. Second, the study had a small sample and relatively high attrition rate, yet the attrition rate was not disproportional across the two arms in each sample. Even with a fixed analysis plan, the fact that the studies were underpowered increases the risk for a spurious relationship between treatment and outcome. Future replication studies and meta-analyses are needed to establish whether found effects were inflated or deflated. ${ }^{37}$ Third, the government did not employ the community-based counsellors as they are not part of the exiting health system, which in a classic pragmatic trial should be the case. Fourth, as mentioned above we failed to reach the target sample size.

A strength of this study is that it combined the evaluation of psychological treatments for both depression and AUD at the same time, reflecting a more real-world integrated care setting. The combination of these disorders represents a large proportion of the global burden caused by mental disorders. Another strength of the study is that it was embedded in a routine, and less strictly controlled, care setting that more closely represent low-resource healthcare settings in most LMICs. This facilitates generalisation of the findings.

The community-based counsellor-delivered HAP is an effective intervention for depression, above and beyond health worker delivered mhGAP-based care (consisting of brief emotional support, psychoeducation and provision of psychotropic medicines when indicated), which is sustained and increased at 12 months - demonstrating long term benefits. For AUD treatment, while a trend for better results among those that participated in the counsellordelivered CAP intervention, this study could not confirm the added value. For governments and organizations working on the integration of treatment for depression into primary health care, this study provides support for the inclusion of psychological treatments as part of the roll out of mhGAP guidelines provided by dedicated community-based counsellors.

The study has several implications. First, it shows that community-based counsellors delivering psychological treatment adds to the effectiveness of depression care within a routine primary healthcare in a low-resource setting. In response to the recent Lancet Commission's question as to how best to deploy psychological treatment in LMICs, this study provides evidence for a delivery model of brief and efficacious psychological treatment by community counsellors in a LMIC setting. ${ }^{7}$ Second, when following mhGAP guidelines for the integration of mental health into primary healthcare, it is therefore important to plan and allocate resources for dedicated psychological workers, such as counsellors, within the government health system. It is important to note that although the community-based counsellors in this study are a relatively low-cost cadre of workers, they received substantial base training and ongoing supervision, which might be an important ingredient in explaining the positive results. Third, future implementation and evaluation of CAP should possibly be restricted to people with harmful alcohol use and not include alcohol dependence. This involves further differentiation of the MHCP to ensure that health workers can distinguish between different degrees of severity of AUD and that additional treatment for people with alcohol dependence is also available.

Mark J. D. Jordans, PhD (iD, Reader, Centre for Global Mental Health, Institute of Psychiatry, Psychology and Neuroscience, King's College London, UK; and Transcultural Psychosocial Organization, Nepal; Nagendra P. Luitel, MA, Researcher, Transcultural Psychosocial Organization, Nepal; Emily Garman, MSC, Researcher, Alan J Flisher Centre for Public Mental Health, Department of Psychiatry and Mental Health, University of Cape Town, South Africa; Brandon A. Kohrt, MD, PhD, Associate Professor, Department of Psychiatry, George Washington University; and Transcultural Psychosocial Organization, Nepal; Sujit D. Rathod, PhD, Assistant Professor, Department of Population Health, London School of Hygiene and Tropical Medicine, UK:

Pragya Shrestha, MA, Clinician, Transcultural Psychosocial Organization, Nepal; Ivan H. Komproe, PhD, Professor, Research and Development Department, HealthNet TPO; and Faculty of Social and Behavioural Sciences, Utrecht University, The Netherlands; Crick Lund, PhD (D), Professor, Alan J Flisher Centre for Public Mental Health,

Department of Psychiatry and Mental Health, University of Cape Town, South Africa; and Centre for Global Mental Health, Institute of Psychiatry, Psychology and Neuroscience, King's College London, UK; Vikram Patel, MD, PhD, Professor, Department of Global Health and Social Medicine, Harvard Medical School; and Department of Global Health and Population, Harvard TH Chan School of Public Health, US

Correspondence: Mark J. D. Jordans, King's College London, Institute of Psychiatry, Psychology and Neuroscience, 16 De Crespigny Park, Camberwell, London SE5 8AF, UK. Email: mark.jordans@kcl.ac.uk

First received 12 Apr 2018, final revision 3 Nov 2018, accepted 9 Dec 2018

\section{Supplementary material}

Supplementary material is available online at https://doi.org/10.1192/bjp.2018.300.

\section{Funding}

This document is an output from the Programme for Improving Mental Health Care Research Programme Consortium, funded by the UK Department of International Development for the benefit of LMICS. B.A.K. Was supported by the US National Institutes of Health (K01MH104310). No funding bodies had any role in study design, data collection and analysis, decision to publish or preparation of the manuscript. The views expressed in this publication are not necessarily those of the funders.

\section{Acknowledgements}

We thank the Transcultural Psychosocial Organization Nepal research teams in Chitwan for their work on this study.

\section{References}

1 Whiteford HA, Degenhardt L, Rehm J, Baxter AJ, Ferrari AJ, Erskine HE, et al. Global burden of disease attributable to mental and substance use disorders: findings from the Global Burden of Disease Study 2010. Lancet 2013; 382: 1575-86.

2 Wang PS, Aguilar-Gaxiola S, Alonso J, Angermeyer MC, Borges G, Bromet EJ, et al. Use of mental health services for anxiety, mood, and substance disorders in 17 countries in the WHO world mental health surveys. Lancet 2007; 370: 841-50.

3 Thornicroft G, Chatterji S, Evans-Lacko S, Gruber M, Sampson N, Aguilargaxiola $\mathrm{S}$, et al. Undertreatment of people with major depressive disorder in 21 countries. Br J Psychiatry 2017; 210: 119-24. 
4 Patel V, Chisholm D, Parikh R, Charlson FJ, Degenhardt L, Dua T, et al. Addressing the burden of mental, neurological, and substance use disorders: key messages from Disease Control Priorities. Lancet 2016; 387: P1672-85.

5 singla DR, Kohrt BA, Murray LK, Anand A, Chorpita BF, Patel V. Psychological treatments for the world: Lessons from low-and middle-income countries. Annu Rev Clin Psychol 2017; 13: 149-81.

6 van Ginneken N, Tharyan P, Lewin S, Rao GN, Meera SM, Pian J, et al. Nonspecialist health worker interventions for the care of mental, neurological and substance-abuse disorders in low- and middle-income countries. Cochrane Database of Systematic Reviews 2013; 11: CD009149.

7 Holmes EA, Ghaderi A, Harmer CJ, Ramchandani PG, Cuijpers P, Morrison AP, et al. The Lancet Psychiatry Commission on psychological treatments research in tomorrow's science. Lancet Psychiatry 2018; 5: 237-86.

8 World Health Organization (WHO). mhGAP Intervention Guide for Mental, Neurological and Substance use Disorders in Non-Specialized Health Settings (Version 2.0). World Health Organization, 2016.

9 Luitel NP, Jordans MJD, Adhikari A, Upadhaya N, Hanlon C, Lund C, et al. Mental health care in Nepal: current situation and challenges for development of a district mental health care plan. Confl Health 2015; 9: 3.

10 Lund C, Tomlinson M, De Silva M, Fekadu A, Shidhaye R, Jordans M, et al. PRIME: A programme to reduce the treatment gap for mental disorders in five low- and middle-income countries. PLOS Med 2012; 9: e1001359.

11 Jordans MJD, Luitel NP, Pokhrel P, Patel V. Development and pilot testing of a mental healthcare plan in Nepal. Br J Psychiatry 2016; 208: s21-8.

12 Luitel NP, Jordans MJD, Kohrt BA, Rathod S, Komproe I. Treatment gap and barriers for mental health care: a cross-sectional community survey in Nepal. PloS one 2017; 12: e0183223.

13 Rathod SD, De Silva MJ, Ssebunnya J, Breuer E, Murhar V, Luitel NP, et al. Treatment contact coverage for probable depressive and probable alcohol use disorders in four low-and middle-income country districts: the PRIME crosssectional community surveys. PloS one 2016; 11: e0162038.

14 Jordans MJD, Kohrt BA, Luitel NP, Komproe IH, Lund C. Accuracy of pro-active case finding for mental disorders by community informants in Nepal. $\mathrm{Br} \mathrm{J}$ Psychiatry 2015; 207: 1-6.

15 Jordans MJD, Luitel NP, Kohrt BA, Lund C, Komproe I. Proactive community case finding to facilitate treatment seeking for mental disorders, Nepal. Bull World Health Org 2017; 95: 531-6.

16 Baron EC, Rathod SD, Hanlon C, Prince M, Fedaku A, Kigozi F, et al. Impact of district mental health care plans on symptom severity and functioning of patients with priority mental health conditions: the Programme for Improving Mental Health Care (PRIME) cohort protocol. BMC Psychiatry 2018; 18: 61.

17 Chowdhary N, Anand A, Dimidjian S, Shinde S, Weobong B, Balaji M, et al. The Healthy Activity Program lay counsellor delivered treatment for severe depression in India: systematic development and randomised evaluation. $\mathrm{Br}$ JPsychiatry 2016: 208: 381-8.

18 Nadkarni A, Velleman R, Dabholkar H, Shinde S, Bhat B, McCambridge J, et al. The systematic development and pilot randomized evaluation of counselling for alcohol problems, a lay counselor-delivered psychological treatment for harmful drinking in primary care in India: The PREMIUM Study. Alcoholism 2015; 39: $522-31$

19 Jordans MJD, Tol WA, Sharma B, van Ommeren M. Training psychosocial counselling in Nepal: content review of a specialised training programme. Intervention 2003; 1: 18-35.

20 Patel V, Weobong B, Weiss HA, Anand A, Bhat B, Katti B, et al. The Healthy Activity Program (HAP), a lay counsellor-delivered brief psychological treatment for severe depression, in primary care in India: a randomised controlled trial. Lancet 2017; 389: P176-85.
21 Weobong B, Weiss HA, MCDaid D, Singla DR, Hollon SD, Nadkarni A, et al. Sustained effectiveness and cost-effectiveness of the Healthy Activity Programme, a brief psychological treatment for depression delivered by lay counsellors in primary care: 12-month follow-up of a randomised controlled trial. PLOS Med 2017; 14: e1002385.

22 Nadkarni A, Weobong B, Weiss HA, McCambridge J, Bhat B, Katti B, et al. Counselling for Alcohol Problems (CAP), a lay counsellor-delivered brief psychological treatment for harmful drinking in men, in primary care in India: a randomised controlled trial. Lancet 2017; 389: P186-95.

23 Nadkarni A, Weiss HA, Weobong B, McDaid D, Singla DR, Park AL, et al. Sustained effectiveness and cost-effectiveness of Counselling for Alcohol Problems, a brief psychological treatment for harmful drinking in men, delivered by lay counsellors in primary care: 12-month follow-up of a randomised controlled trial. PLOS Med 2017; 14: e1002386.

24 Gilbody S, Richards D, Brealey S, Hewitt C. Screening for depression in medical settings with the Patient Health Questionnaire (PHQ): a diagnostic meta-anaIysis. J Gen Intern Med 2007; 22: 1596-602.

25 Kohrt BA, Luitel NP, Acharya P, Jordans MJD. Detection of depression in low resource settings: validation of the Patient Health Questionnaire (PHQ-9) and cultural concepts of distress in Nepal. BMC Psychiatry 2016; 16: 58

26 Saunders JB, Aasland OG, Babor TF, De La Fuente JR, Grant M. Development of the alcohol use disorders identification test (AUDIT): WHO collaborative project on early detection of persons with harmful alcohol consumption-II. Addiction 1993; 88: 791-804.

27 Pradhan B, Chappuis F, Baral D, Karki P, Rijal S, Hadengue A, et al. The alcohol use disorders identification test (AUDIT) : validation of a Nepali version for the detection of alcohol use disorders and hazardous drinking in medical settings. Subst Abuse Treat Prev Policy 2012; 7: 42.

28 Üstün TB, Kostanjsek N, Chatterji S, Rehm J. Measuring Health and Disability: Manual for WHO Disability Assessment Schedule WHODAS 2.0. World Health Organization, 2010.

29 Luitel N, Jordans M, Murphy A, Roberts B, McCambridge J. Prevalence and patterns of hazardous and harmful alcohol consumption assessed using the AUDIT among Bhutanese refugees in Nepal. Alcohol Alcohol 2013; 48: 349-55.

30 Tol WA, Komproe IH, Thapa SB, Jordans MJD, Sharma B, de Jong JTVM. Disability associated with psychiatric symptoms among torture survivors in rural Nepal. J Nerv Ment Dis 2007; 195: 463-9.

31 Richardson DB, Kinlaw AC, MacLehose RF, cole SR. Standardized binomial models for risk or prevalence ratios and differences. Int J Epidemiol 2015; 44: 1660-72.

32 Hernán M, Robins J. Causal Inference. CRC Press, 2018

33 Sullivan GM, Feinn R. Using effect size-or why the P value is not enough. $J$ Grad Med Edu 2014; 4: 279-82.

34 Kirkwood B, Sterne J. Essential Medical Statistics. Blackwell Science, 2003.

35 Bertholet N, Daeppen JB, Wietlisbach V, Fleming M, Burnand B. Reduction of alcohol consumption by brief alcohol intervention in primary care: systematic review and meta-analysis. Arch Intern Med 2005; 165: 986-95.

36 Jordans MJD, Aldridge L, Luitel NP, Baingana F, Kohrt BA. Evaluation of outcomes for psychosis and epilepsy treatment delivered by primary health care workers in Nepal: a cohort study. Int J Ment Health Syst 2017; 11: 70.

37 Ioannidis JPA. Why most discovered true associations are inflated. Epidemiology 2008: 640-8. 